\title{
AUTOMATED SEMMES WEINSTEIN MONOFILAMENT EXAMINATION REPLICATION USING OPTICAL IMAGING AND MECHANICAL PROBE ASSEMBLY
}

\author{
Hafeez U.R, Siddiqui, Stephen R. Alty (SMIEEE, Michelle Spruce, and Sandra Dudley (MIEEE)
}

\author{
School of Engineering, London South Bank University, 103 Borough Road, London, SE1 0AA, U.K \\ (e-mail: siddiqh3@lsbu.ac.uk and dudleyms@lsbu.ac.uk).
}

\begin{abstract}
The World Health Organization reports more than 135 million people globally suffer from diabetes, with $25 \%$ developing peripheral neuropathy and estimates the numbers living with diabetes will reach over 300 million by 2025 . Peripheral neuropathy is a term used to describe the loss of feeling in the peripheral limbs. If not properly managed, amputation of the lower limbs can be the result. Regular screening is required for this condition so as to avoid further deterioration. This paper describes an automated peripheral neuropathy testing device replicating the widely accepted Semmes Weinstein Monofilament Examination. In this paper a patient's foot is scanned optically and the subsequent image processing and grid information algorithms presented reliably identify the plantar surface sensory neuropathy pressure points on a given patient's foot. Then, these coordinates are relayed to an automated mechanical probe driven by a microcontroller where it randomly applies the accepted $98 \mathrm{mN}(10 \mathrm{~g})$ of force to those pressure points.
\end{abstract}

Index Terms - Optical Imaging, Foot Sectorization, Plantar Surface, Neuropathy, Diabetes, Binary Image, Contours, Skin Tone, Microcontroller

\section{INTRODUCTION}

Diabetic peripheral neuropathy (DPN) affects up to onethird of adults with diabetes [1] and is considered one of the most destructive forms of $\mathrm{PN}$ encountered by orthopedic surgeons [1]. Most types of PN cause pain or impaired sensation in limb extremities, and when presented in diabetic patients it often leads to the development of foot ulcers, infections, and neuropathic arthropathy, with subsequent deformity, slow healing of lower extremity wounds and injuries. Foot ulcers, infections, and deformities are some of the biggest reasons for morbidity and unfortunately subsequent mortality rates amongst the diabetic population.

Approximately $60 \%$ of diabetic patients develop one or several of these neuropathies within 10 years following diagnosis [2]. In 2000, numbers suffering already reached 171 million with predictions sufferers will exceed 380 million by 2025 [3]. At any point in time, $3 \%$ to $4 \%$ of the diabetic population will have a foot ulcer or infection. Fifteen percent of individuals living with diabetes will have a foot ulcer in their lifetime, and foot ulcers precede $85 \%$ of lower extremity amputation in the diabetic patient population. In 2004 there were 71,000 lower extremity amputations in diabetic patients in the United States alone. Eighty-five percent were preceded by diabetic foot ulcer [1]. Once amputated, the 2-years mortality has been reported to be as high as $36 \%$. These patients are also at a greater risk of premature death, even if they do not undergo an amputation [1]. The rate of lower limb amputation in diabetic patients is $10-30$ times higher than that for nondiabetics generally and an average of one leg is amputated every 30 seconds worldwide. There is a $50 \%$ risk of a second amputation during the first two years following the first amputation and 50\% of patients may die in the three years following a lower limb amputation [3]. Currently approximately 3.8 million people suffer from diabetes in the United Kingdom and numbers are expected to double by 2035/6 [2]. The cost reported in 2010/2011 in UK was $£ 23.7$ billion of which $£ 9.8$ billion was direct costs and $£ 13.9$ billion indirect costs with costs increasing to an estimated $£ 39.8$ billion by 2035/2036 [4]. Recognition of condition changes and deterioration is crucial for preventive strategies and these have been demonstrated to decrease the potential risk for the development of diabetic foot ulcers, foot infection, Charcot foot, or amputation [1]. Diabetic neuropathy consists of multiple manifestations, of which loss of sensation is most prominent.

The Semmes Weinstein Monofilament Examination (SWME) method is currently one of the most practiced manual screening approaches for clinically significant neuropathy and its effectiveness has been evaluated by researchers [5]. This SWME involves a trained podiatrist testing a patient's foot with a hand held nylon monofilament probe over five specific pressure points in three plantar surface areas, the toe, metatarsal and heel. The nylon probe is designed to bend $10 \mathrm{~mm}$ when $10 \mathrm{~g}(98 \mathrm{mN})$ force is applied to each of these five points. The inability of a patient to detect the above force on any of the tested 
pressure points may mean that the area in question is an insensate area and possible PSN should be further investigated. The SWME technique is simple and the most widely used but it can be considered cumbersome, laborintensive, repeatability is difficult to maintain and result outcomes are prone to experimenter bias especially since the $10 \mathrm{~mm}$ bend is judged by eye in most cases [6].

This paper describes a new approach to automatically replicate the SWME method to select the five suitable pressure test points along the plantar surface pressure regions, namely the toe (hallux), metatarsal heads and heel (calcaneum), on a given patient's foot. The procedure uses optical image processing incorporating grid information. Previous work presented by the authors covered pressure points detection of the plantar contact area using optical imaging of the complete foot [7]. Here, the system assembly remains the same, but, the authors concentrate on a computational grid imaging technique combined with an internal mechanical probe producing an automated SWME system. Previously only the specific color of the plantar surface in greater contact with the scanner was used and the approach was considered non-generic [7].

The previous algorithm identified three separate sectors, one for each pressure area used by the SWME method. Each area was then automatically inferred by its size i.e. the largest rectangle would contain heel area and the smaller rectangle would hold the toe area. The previous algorithm wasn't suitable for participants with flat-feet, for example as their entire foot applied similar pressure to the scanner, thus one large rectangle could result, making area discrimination difficult. In the current approach, skin color and a wider database of human skin color set is used to detect the entire foot in a single rectangle, and then extract pressure points followed by using the grid information to localize the pressure regions. Hence the current approach is more generic and can accommodate flat and non-flat feet as well as different ethnicities. Grid sectorization enables faster pressure point recognition.

\section{METHODOLOGY}

We first describe the optical imaging technique followed by the mechanical probe execution. The foot image is scanned using a flatbed scanning technique and the obtained image is shown in Fig. 1(a). The in-house designed image processing code extracts the object (foot) from the background (image) and draws a border around the detected foot as shown in Fig. 1(b). The obtained foot image (object) is then sub-divided into a fixed dimensions of grid as shown in Fig. 1(c). The space, cell size of the grid, amongst grid rows and columns is correlated to the size of the foot being scanned.

Following the grid incorporation, two computational phases are then performed.

In phase 1, the foot image is sub-sectioned into approximated pressure regions of interest using the grid information. The sub-sectioned regions are namely the toe, metatarsal, and heel regions.

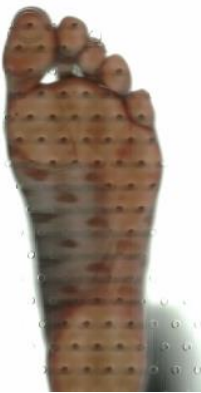

(a)

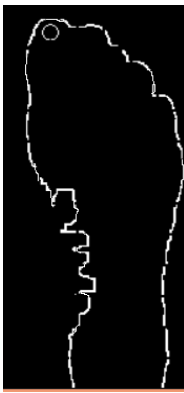

(b)

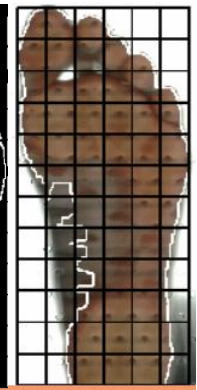

(c)
Fig. 1. Processing the input image (a): input image. (b): External boundary (c): Foot in Grid

For a better understanding, an analogy of matrix element position and grid cell position is made, hence the entire grid is represented by a matrix ' $G$ ' as given below.

$$
\mathrm{G}=\left[\mathrm{g}_{\mathrm{i}, \mathrm{j}}\right]_{\mathrm{M} \times \mathrm{N}}
$$

here, ' $g$ ' represents the subelement, (grid cell) within the grid $\mathrm{G}, \mathrm{i}$ and $\mathrm{j}$ are cell position within the rows $\mathrm{M}$ and columns $\mathrm{N}$ respectively, where $1 \leq \mathrm{i} \leq \mathrm{M}$ and $1 \leq \mathrm{j} \leq \mathrm{N}$ and $\mathrm{M}=12$ and $\mathrm{N}=6$. For example the first top left cell in the grid is represented by $\mathrm{g}_{1,1}$ and the 2 nd cell in first row is represented by $\mathrm{g}_{1,2}$ and so on.

The region of interest (ROI) for the toe pressure area always lies in a sub-rectangle or sub-matrix ' $\mathrm{T}$ ' i.e. $\mathrm{T} \subseteq \mathrm{G}$; $\mathrm{T}=\left[\mathrm{t}_{\mathrm{i}, \mathrm{j}}\right]_{2 \times 2}$

where $1 \leq \mathrm{i} \leq 2$ and $1 \leq \mathrm{j} \leq 2$, where ' $\mathrm{t}$ ' represents one cell inside the $T$, subset of $G$, as shown in Fig. 2. The element of matrix, grid, $\mathrm{T}$ are $\left(\mathrm{t}_{1,1}, \mathrm{t}_{1,2}, \mathrm{t}_{2,1}, \mathrm{t}_{2,2}\right)$.

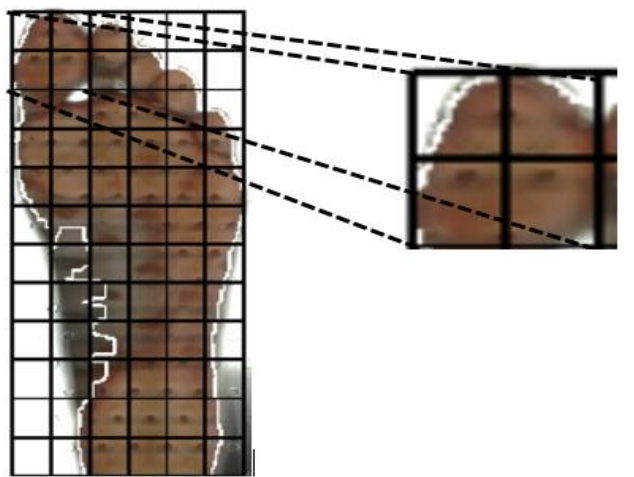

Fig. 2. Extraction of toe area using grid

The metatarsal area is then computed so as to correctly identify the pressure points for this region. Using the same matrices analogy, the metatarsal area lies at fore foot minus toe and it always resides in grid area, represented by a matrix ' $\mathrm{M}_{\mathrm{e}}$ ' as follows: 


$$
\mathrm{M}_{\mathrm{e}}=\left[\mathrm{m}_{\mathrm{i}, \mathrm{j}}\right]_{4 \times 6}
$$

where $3 \leq \mathrm{i} \leq 6$ and $1 \leq \mathrm{j} \leq 6$ and $\mathrm{Me} \subseteq \mathrm{G}$ and ' $\mathrm{m}$ ' represent one cell inside the subset ' $\mathrm{M}_{\mathrm{e}}$ ' with relative position of ith row and jth column in $\mathrm{G}$ as shown in Fig. 3(a).

To avoid the mechanical probe hitting the outer edge of a patient's foot, the heel area pressure point is defined by subtracting one grid row from the max y-coordinate of the external boundary contour. The heel area always lies in the grid area, represented by a matrix, grid ' $\mathrm{H}$ ' as follows:

$$
\mathrm{H}=\left[\mathrm{h}_{\mathrm{i}, \mathrm{j}}\right]_{2 \times 4}
$$

where $11 \leq \mathrm{i} \leq 12$ and $2 \leq \mathrm{j} \leq 5$ and $\mathrm{H} \subseteq \mathrm{G}$ and ' $\mathrm{h}$ ' represent one cell inside the subset $\mathrm{H}$ with relative position of ith row and jth column in $\mathrm{G}$ as shown in Fig. 3(b).

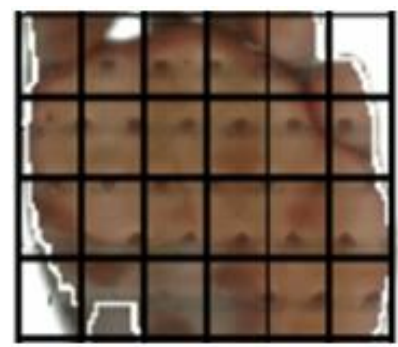

(a)

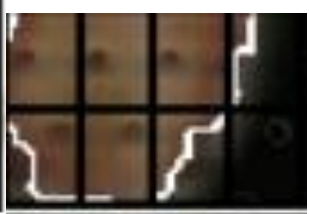

(b)
Fig. 3. Sectorization of metatarsal area (a) and Heel area (b) by dissecting grid

The grid formation is used in phase 1 to dissect the foot image further and to produce areas that represent the plantar surface pressure points in the regions found above. Using the grid in this phase enables a faster processing time as it promotes a more accurate sub-detection of the pressure region via the grid and excludes regions outside the area of interest.

In the second phase, grid panels (cells) identified specifically within the 3 pressure areas found in phase 1 above are then used to identify the appropriate pressure points within each region as follows. The subsequent algorithm then considers only those contour points of the toe that lie within this sub-rectangle T. A central point of the toe polygon is obtained by applying trapezoidal centroid.

The algorithm is then applied on the sub rectangle $\mathrm{M}$ and three pressure points, the first metatarsophalangeal joint and a central point between these two points are found, using the extreme contour points on the $\mathrm{x}$-axis.

The pressure point at the heel is extracted by subtracting one grid step from the y-coordinate of the contour's point that has the largest y-coordinates. Results for 9 participants are shown in Fig. 4. Participant details are presented in Table's 1 and 2.

Investigations to optimize the grid size were performed and it was established that that the performance of the grid increased and peaked when it had dimensions of 12 rows and 6 columns, with further division of the grid deteriorating the performance. This is to say that the grid is optimized as above, but the cell size of the grid varies with foot size. The grid information successfully classifies the pressure regions i.e. the accepted toe, metatarsal and heel areas.

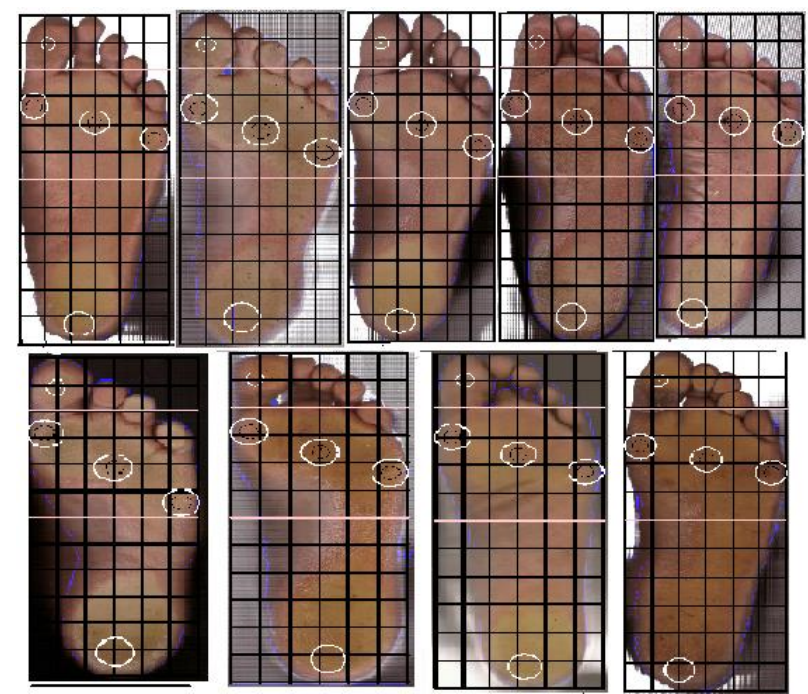

Fig. 4. Foot images with grid of 12 rows and 6 column and extracted pressure points.

Subsequently, the mechanical phase takes place. The location of the extracted pressure points are then translated in terms of rows and columns position which are then aligned to a hole in the perforated sheet closest to the chosen pressure point. This information is then relayed to an off the shelf microcontroller (MC) board via serial communication. A schematic of the overall automated SWME sequence is shown in Fig. 5 and is explained as follows.

A probe assembly was fabricated in-house using precision components and a commercial amplifier. The system was then calibrated to apply exactly $98 \mathrm{mN} \pm 1 \%$. This assembly is driven by stepper motor controlled rails in both the $\mathrm{X}$ and $\mathrm{Y}$-axes. Once the microcontroller has received the correct coordinates from the algorithms as described it directs the probe assembly accordingly, a further Z-axis stepper motor is then used to drive the probe onto the plantar surface to apply exactly $98 \mathrm{mN}$ to each site in turn. If the patient feels the probe, they record their response by pressing a handheld button which is wired into the microcontroller. The response latency is recorded by the microcontroller and a two second window is permitted for a positive response to be recorded. Furthermore, a random delay and probe sequence is introduced before the probe is activated to mitigate false positives or "guessing" by the patient. Once all five test sites have been examined, the microcontroller returns the probe to its home location and transfers the results of the tests to the host personal computer (PC). 


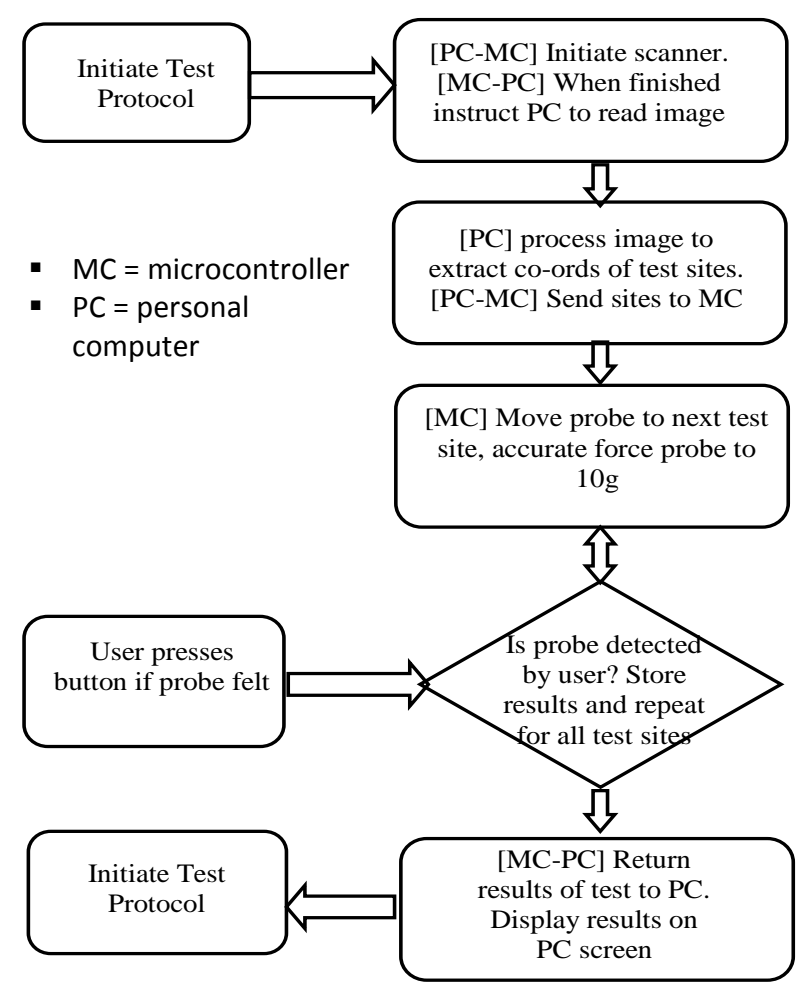

Fig. 5. Event sequence for overall SWME replication.

\section{RESULTS}

This set-up was not a clinical trial so the participants were limited to healthy subjects, future blind tests will include diabetic subjects. The database consists of 9 images of subjects from different ethnicity, age and gender demographics, as shown in table 1 and table 2. A total of 6 males and 3 females volunteered, the mean age of the subjects being $33.33 \pm 26.7$ years. The algorithm presented showed a $100 \%$ success rate.

TABLE 1.
\begin{tabular}{|c|c|}
\hline Age Group & Quantity \\
\hline $20-25$ & 1 \\
\hline $25-30$ & 1 \\
\hline $30-35$ & 2 \\
\hline $35-40$ & 3 \\
\hline $40-50$ & 1 \\
\hline $50-70$ & 1 \\
\hline
\end{tabular}

1. Age group

TABLE 2.
\begin{tabular}{|l|c|}
\hline Ethnicity & Quantity \\
\hline Western European & 2 \\
\hline Eastern European & 2 \\
\hline African & 2 \\
\hline Asian & 3 \\
\hline Total & 9 \\
\hline 2. Ethnicities
\end{tabular}

\section{CONCLUSION}

This paper presents an automated replication of the Semmes Weinstein Monofilament Examination to perform pressure points detection for peripheral neuropathy assessment of a diabetic patient's plantar surface using an optical imaging incorporation with grid information. The proposed method extracts the outer boundary followed by optical foot detection. The pressure region is then classified using a grid based technique. The required pressure points are then obtained by considering the contour points lying in approximated pressure region. The algorithm was successfully applied on 9 users. Further research focuses on lesion recognition on the plantar surface to avoid the probe application on pressure points where they overlap a lesion.

\section{ACKNOWLEDGMENT}

Mr. Siddiqui's partial PhD Scholarship, won by Dr Dudley is sponsored by London South Bank University. The team also thank Mr. Peter Hadfield for his assistance.

\section{REFERENCES}

[1] M. S. Pinzur, "Diabetic Peripheral Neuropathy," Foot and Ankle Clinics, 16(2), ELSEVIER, Chicago, pp. 345-349, June, 2011.

[2] NHS, "Failings in diabetes care 'cost thousands of lives," nhs.co.uk. Nov. 2012. Available from: http://www.nhs.uk/news/2012/11November/Pages/Failingsin-diabetes-care-cost-thousands-of-lives.aspx [Accessed $10 / 22 / 13]$.

[3] M. Aalaa, O. T. Malazy, M. Sanjari, M. Peimani and M. Tehrani, "Nurses' role in diabetic foot prevention and care; a review," Journal of Diabetic \& Metabolic Disorders [Online]. Vol 11, pp. 2-6, Nov. 2012. Available: http://www.jdmdonline.com/content/pdf/2251-6581-1124.pdf

[4] G. Bove, "Mechanical sensory threshold testing using nylon monofilaments: The pain field's 'Tin Standard' " journal of the International Association for the Study of Pain, Department of Anesthesia and Critical Care, Beth Israel Deaconess Medical Center and Harvard Medical School, pp 13-17, 2006.

[5] N. Kamei, K. Yamane, S. Nakanishi, Y. Yamashita, T. Tamura, K. Ohshita, H. Watanabe, R. Fujikawa, M. Okubo, N. Kohno, "Effectiveness of Semmes-Weinstein Monofilament Examination for diabetic peripheral neuropathy screening,”. NCBI, ELSEVIER. [Online]. 19(1), pp. 47-53, Jan. $2005 . \quad$ Available: http://www.ncbi.nlm.nih.gov/pubmed/15642490

[6] N. Hex, C. Bartlett, D. Wright, M. Taylor, and D. Varley, "Estimating the current and future costs of Type 1 and Type 2 diabetes in the UK, including direct health costs and indirect societal and productivity costs," Diabetic Medicine, 29(7), pp 855-862, June, 2012.

[7] H. R. Siddiqui, S. R. Alty, S. M. Spruce, Sandra E. Dudley, "Automated Peripheral Neuropathy Assessment of Diabetic Patients using Optical Imaging and Binary Processing Techniques," International IEEE Conference on IEEE Pointof-Care Healthcare Technologies (PHT), Bangalore, India. pp. 200-203, January 2013. 